\title{
Comparative Study between Betamethasone and Dexamethasone as a Prophylactic Corticosteroids Therapy on Neonatal Outcome in Elective Cesarean Section at 37 Weeks
}

\author{
Abdalla Khalil Issa ${ }^{1}$, Wael Soliman Taha ${ }^{1}$, Abdelsattar Abdullah Elsayeh ${ }^{2}$, Hany Khayri Elhadad ${ }^{1}$ \\ 1-Obstetrics and Gynecology Department, 2- Pediatrics Department, Faculty of Medicine, Al Azhar \\ University
}

*Corresponding Author: Hany Khayri Elhadad, Phone No.: (+2) 01025017934, E-mail: hanykh123@yahoo.com

\begin{abstract}
Background: preterm birth is the most costly complication of pregnancy and the leading cause of neonatal morbidity and mortality. There are multiple strategies to minimize the risk and the impact of prematurity, such as administration of antenatal corticosteroids (ACS), in association with tocolysis, neuroprotection with magnesium sulphate, and neonatal life-saving therapies. Aim of the Work: this study aimed to assess the comparison between betamethasone and dexamethasone before elective cesarean section at (37-37+6) weeks, in reducing neonatal respiratory morbidity and admission to neonatal intensive care unit (NICU) with respiratory complications. Patients and Methods: this clinical observational prospective study had been conducted at El Hussein University Hospitals Obstetrics and Gynecology Department from December 2017 to July 2018 to assess the comparison between betamethasone and dexamethasone as a prophylactic corticosteriodes before elective cesarean section at (37-37+6) weeks. Five hundred pregnant women attended to out-patient clinic, the patient were at risk of preterm labour before 37 weeks. Results: the rate of neonatal admission to primary care baby unit (PCBU) and TTN was significantly lower in group I [Betamethasone Group] when compared to group II [Dexamethasone Group]. There was a statistically significant difference between both groups regarding need for mechanical ventilation ( 1 to 9 ) respectively with $\mathrm{p}$ value of 0.011 .Conclusion: antenatal corticosteroids (ecpecially betamethasone) were effective in prevention of respiratory complications after elective caesarean section at term.
\end{abstract}

Keywords- Betamethasone, Dexamethasone, Cesarean Section

\section{INTRODUCTION}

Antenatal corticosteroid treatment of women at risk of very preterm birth before 34 weeks gestation is one of the most effective treatment for the prevention of RDS, reducing neonatal mortality and morbidity ${ }^{(\mathbf{1})}$. This is of importance for developing countries where resources are scarce and it is often difficult to provide expensive treatment such as neonatal care. Based on high grade evidence, antenatal steroid therapy is very effective in preventing neonatal mortality and morbidity, yet remains at low coverage in low/middle-income countries. If scaled up, this intervention could save up to 500000 neonatal lives annually (2).Pulmonary surfactant is produced by type II alveolar cells. It spreads in the lung tissue preventing alveolar collapse during expiration and the alveoli to open easily at the next inspiration (1). Antenatal corticosteroids administration to promote fetal lung maturity is the one of the most effective interventions to prevent the morbidity and mortality associated with prematurity as the cause premature liberation of surfactant from the alveoli perhaps by induction of an enzyme concerned with the biosynthesis of surfactant. The steroids used are fluorinated steroids, with identical biological activity and readily cross the placenta in their biological active forms ${ }^{(3)}$. Betamethasone has a longer half-life than dexamethasone and the interval from treatment to delivery may have impacted efficacy of steroids. Betamethasone administration for pregnant women who are at risk of premature delivery results in more substantial reduction in the risk of respiratory distress syndrome (RDS), intraventricular hemorrhage (IVH) and retinopathy of prematurity ${ }^{(4)}$. Synthetic glucocorticoids have been successfully employed for more than 20 years to enhance fetal lung maturation in situations where preterm delivery is anticipated. The most extensively studied regimens of corticosteroid treatment are two doses of betamethasone $12 \mathrm{mg}$ given intramuscularly 24 
hours apart or four doses of dexamethasone 6 mg given intramuscularly 12 hours (5). Antenatal steroids for term caesarean section randomised trials are sought to evaluate whether giving the recommended dose of betamethasone before delivery may lead to a reduction in respiratory morbidity in babies delivered by section at term ${ }^{(4)}$.Betamethasone given before elective caesarean section at term reduces respiratory distress and admission to a special care baby unit. The benefit falls with increasing gestation, supporting the recommendation to delay elective caesarean section until the 39th week. Nevertheless, the benefits of antenatal steroids persist till 39 weeks ${ }^{(6)}$.

\section{AIM of the WORK}

The objective of this study was to assess the comparison between betamethasone and dexamethasone before elective cesarean section at (37-37+6)weeks, in reducing neonatal respiratory morbidity and admission to neonatal intensive care unit (NICU) with respiratory complications.

\section{PATIENTS and METHODS}

This clinical observational prospective study had been conducted at El Hussein University Hospitals Obstetrics and Gynecology department from December 2017 to July 2018. The study was approved by the Ethics Board of Al-Azhar University.

To assess the comparison between betamethasone and dexamethasone as a prophylactic corticosteriodes before elective cesarean section at $(37-37+6)$ weeks. Five hundred pregnant women attended to outpatient clinic, the patient were at risk of preterm labour before 37 weeks with the follwoing.

\section{Inclusion criteria:}

- Maternal age between $20 \mathrm{y}$ and $40 \mathrm{y}$.

- Previous caesarean section at (37-37+6 weeks) of pregnancy as calculated from the $1^{\text {st }}$ day of the LMP.

- Previous caesarean section.

- Pregnant women undergoing elective caesarean delivery due to absolute obstetric indication for caesarean section.

- Patient at risk of preterm birth such as:

- Histoty of previous preterm birth.

- History of recurrent spontaneous abortion.

○ Polyhydroaminious.
- History of Premature rupture of membranes

Exclusion criteria:

1. Any associated medical problem with pregnancy (Septicemia, Maternal diabetes, Chronic hypertension Chronic renal diseases, Liver diseases).

2. Women on corticosteroids treatment for anather disease.

3. Multiple gestations.

4. Known infant with structural malformation.

Five hundred pregnant women at risk of preterm labour before 37 weeks divided into tow groups as the following:

- Group A:

250 women with singleton pregnancies was asked to participate in the study and received betamethasone $14 \mathrm{mg}$ I.M on 2 consecutive days 48 hours before the elective caesarian section. The elective CS was done at (37-37+6wks) (betafose injection - Amon pharm company).

- Group B:

250 women with singleton prégnances were asked to participate in this study received three intramuscular doses of $8 \mathrm{mg}$ dexamethazone 8 hours apart 48 hours before the elective caesarian section. The elective CS was done at (37-37+6wks) (Dexamethasone injection -Amriya pharm company).

All patient enrolled in this study subjected to following after giving their oral and written consent:

1. History taking: complete personal and medical history taking with special emphasis on LMPD to determine the exact gestational age.

2. Examination:

- General examination.

- Abdominal examination.

- Local examination: fundal level, fundal grip, pelvic grip and fetal heart sound.

- Assessment of blood pressure.

- Determination of gestational age by the date of last menstrual period and confirmed by first trimester ultrasound.

- Laboratory investigations at the start and one week before termination which including:

- Fasting blood sugar and post prandial blood sugar.

- Renal function tests.

- Total urine analysis. 
Comparative Study between Betamethasone and Dexamethasone as a Prophylactic...

- Serum alanine aminotransferase (ALT).

- Complete blood count (CBC).

- Coagulation profile.

Outcome measures:

The Primary outcome

- Neonatal Respiratory distress:

1. Respiratory distress syndrome:

$\mathrm{RD}$ was defined as presence of at least two of the following Criteria:

i. $\quad$ Tachypnea ( $>60$ breaths per minute).

ii. Central Cyanosis in room air.

iii. Expiratory grunting.

iv. Subcostal, intercostals or jugular retractions and nasal flaring.

2. Transient Tachypnea of the neonate:

Consisted of a period of rapid breathing (higher than the normal range of 40-60 times per minute this condition is self limited and resolves over 24-48 hours.

\section{The Secondary outcomes}

- Development of neonatal respiratory complications (pneumonia, air leak syndrome).

- Perinatal death (within 1st 24 hours).

- Length of stay in the NICU.

- Need for mechanical ventilation.

- Admission of the neonate to special care baby unit (NICU) for all levels of respiratory distress.

The neonate were followed up by recording:

1- The incidence of admission with respiratory distress to neonatal ICU.

2- The incidence of Transient Tachypnea of the newborn.
3- The incidence of respiratory distress syndrome.

4- Need for mechanical ventilation

5- Apgar scoring.

6- Down score for assessment of respiratory distress ${ }^{(7)}$.

\section{7- Level of resuscitation:}

- Routine care of newborn.

- Positive pressure ventilation

\section{Statistical methods}

The collected data were coded, tabulated, and statistically analyzed using IBM SPSS statistics (Statistical Package for Social Sciences) software version 18.0, IBM Corp., Chicago, USA, 2009.

Descriptive statistics were done for quantitative data as minimum \& maximum of the range as well as mean \pm SD (standard deviation) for quantitative normally distributed data, while it was done for qualitative data as number and percentage.Inferential analyses were done for quantitative variables using Shapiro-Wilk test for normality testing, independent $t$-test in cases of two independent groups with normally distributed data. In qualitative data, inferential analyses for independent variables were done using Chi square test for differences between proportions and Fisher's Exact test for variables with small expected numbers. The level of significance was taken at:

- P value $>0.050$ was insignificant.

- P value $<0.050$ was significant.

- P value $<0.001$ was highly significant.

\section{RESULTS}

There was no significant difference between the studied groups regarding maternal characteristics (Table 1).

Table 1: comparison between the studied groups regarding maternal characteristics

\begin{tabular}{|c|c|c|c|c|}
\hline \multicolumn{2}{|c|}{ Variables } & Betamethasone ( $\mathrm{N}=239)$ & Dexamethasone ( $\mathrm{N}=236)$ & $\mathbf{P}$ \\
\hline \multirow{2}{*}{ Age (years) } & Mean \pm SD & $28.4 \pm 3.6$ & $28.0 \pm 3.6$ & \multirow{2}{*}{$\wedge^{\wedge} 0.169$} \\
\hline & Range & $21.0-38.0$ & $21.0-38.0$ & \\
\hline \multirow{3}{*}{ BMI $\left(\mathrm{kg} / \mathrm{m}^{2}\right)$} & Mean \pm SD & $26.9 \pm 2.0$ & $27.1 \pm 1.9$ & \multirow{3}{*}{$\wedge 0.258$} \\
\hline & Range & $21.7-32.9$ & $22.2-32.6$ & \\
\hline & Multi & $76(31.8 \%)$ & $85(36.0 \%)$ & \\
\hline \multirow{4}{*}{$\begin{array}{c}\text { Indications of } \\
\operatorname{CS}(\mathbf{n}, \%)\end{array}$} & Previous CS & $213(89.1 \%)$ & $221(93.6 \%)$ & \multirow{4}{*}{$\& 0.571$} \\
\hline & Malpresentation & $16(6.7 \%)$ & $10(4.2 \%)$ & \\
\hline & Placenta previa & $7(2.9 \%)$ & $2(0.8 \%)$ & \\
\hline & Others & $3(1.3 \%)$ & $3(1.5 \%)$ & \\
\hline
\end{tabular}

${ }^{\wedge}$ Independent t-test, \#Chi square test, \&Fisher's Exact test

There was no significant difference between the studied groups regarding neonatal characteristics (weight and sex) (Table 2).

Table 2: comparison between the studied groups regarding neonatal characteristics 


\begin{tabular}{|c|c|c|c|c|}
\hline \multicolumn{2}{|c|}{ Variables } & Betamethasone (N=239) & Dexamethasone (N=236) & P \\
\hline \multirow{2}{*}{ Weight $(\mathbf{k g})$} & Mean \pm SD & $3.1 \pm 0.3$ & $3.1 \pm 0.2$ & \multirow{2}{*}{0.390} \\
\cline { 2 - 4 } & Range & $2.3-3.7$ & $2.5-3.8$ & \multirow{2}{*}{$\# 0.825$} \\
\cline { 2 - 4 } \multirow{2}{*}{ Sex (n, \%) } & Male & $128(53.6 \%)$ & $124(52.5 \%)$ & $112(47.5 \%)$ \\
\cline { 2 - 4 }
\end{tabular}

${ }^{\wedge}$ Independent t-test, \#Chi square test

There were no significant differences between groups regarding 1-min and 5-min Apgar scores but Neonates of betamethasone group had lower incidence of respiratory distress as evidenced by lower down score table (Table 3).

Table 3: comparison between the studied groups regarding neonatal condition at birth

\begin{tabular}{|c|c|c|c|c|}
\hline \multicolumn{2}{|c|}{ Scores } & Betamethasone ( $\mathrm{N}=239)$ & Dexamethasone $(\mathrm{N}=236)$ & ${ }^{\wedge} \mathbf{P}$ \\
\hline \multirow{2}{*}{ APGAR1minute } & Mean \pm SD & $6.9 \pm 1.0$ & $6.7 \pm 1.2$ & \multirow{2}{*}{0.077} \\
\hline & Range & $4.0-9.0$ & $4.0-9.0$ & \\
\hline \multirow{2}{*}{ APGAR5minute } & Mean \pm SD & $8.3 \pm 1.1$ & $8.1 \pm 1.5$ & \multirow{2}{*}{0.057} \\
\hline & Range & $4.0-10.0$ & $4.0-10.0$ & \\
\hline \multirow{2}{*}{ Down score } & Mean \pm SD & $2.1 \pm 0.9$ & $2.4 \pm 0.9$ & \multirow{2}{*}{$0.001 *$} \\
\hline & Range & $0.0-5.0$ & $0.0-7.0$ & \\
\hline
\end{tabular}

^Independent t-test, $*$ Significant

Neonates of betamethasone group had less frequent neonatal respiratory diseases, but the differences were significant only in TTN and RDS (Table 4).

Table 4: comparison between the studied groups regarding neonatal respiratory diseases

\begin{tabular}{|c|c|c|c|c|c|}
\hline \multicolumn{2}{|c|}{ Diseases } & $\begin{array}{c}\text { Betamethasone } \\
(\mathrm{N}=\mathbf{2 3 9})\end{array}$ & $\begin{array}{c}\text { Dexamethasone } \\
(\mathrm{N}=\mathbf{2 3 6})\end{array}$ & $\mathbf{P}$ & $\begin{array}{c}\text { RR } \\
(95 \% \mathrm{CI})\end{array}$ \\
\hline \multicolumn{2}{|c|}{ TTN } & $4(1.7 \%)$ & $16(6.8 \%)$ & $\# 0.006 *$ & $\begin{array}{c}0.39 \\
(0.16-0.93)\end{array}$ \\
\hline \multicolumn{2}{|c|}{ RDS } & $1(0.4 \%)$ & $7(3.0 \%)$ & \&0.031* & $\begin{array}{c}0.25 \\
(0.04-1.54)\end{array}$ \\
\hline \multirow{2}{*}{ RDS grade } & Mild & $1(100.0 \%)$ & $3(42.9 \%)$ & \multirow{2}{*}{$\& 0.285$} & \multirow[b]{2}{*}{--} \\
\hline & Moderate & $0(0.0 \%)$ & $4(57.1 \%)$ & & \\
\hline \multicolumn{2}{|c|}{ Pneumothorax } & $0(0.0 \%)$ & $2(0.8 \%)$ & $\& 0.246$ & -- \\
\hline
\end{tabular}

\#Chi square test, \&Fisher's Exact test, *Significant, RR: Relative rate, CI: Confidence interval, TTN=transient tachypnea of newborn, RDS=respiratory distress syndrome.

Neonates of betamethasone group had significantly less frequent need for positive resuscitation, NICU admission and MV (Table 5).

Table 5: comparison between the studied groups regarding neonatal interventions

\begin{tabular}{|c|c|c|c|c|c|}
\hline \multicolumn{2}{|c|}{ Interventions } & Betamethasone ( $\mathbf{N}=239)$ & Dexamethasone $(\mathrm{N}=\mathbf{2 3 6})$ & $\mathbf{P}$ & $\begin{array}{c}\text { RR } \\
(95 \% \mathrm{CI})\end{array}$ \\
\hline \multirow{2}{*}{ Resuscitation } & Positive & $6(2.5 \%)$ & $28(11.9 \%)$ & \multirow{2}{*}{$\#<0.001 *$} & \multirow{2}{*}{$0.33(0.16-0.69)$} \\
\hline & Routine & $233(97.5 \%)$ & $208(88.1 \%)$ & & \\
\hline \multicolumn{2}{|c|}{ NICU admission (n, \%) } & $5(2.1 \%)$ & $25(10.6 \%)$ & $\#<0.001 *$ & $0.32(0.14-0.71)$ \\
\hline \multirow{2}{*}{$\begin{array}{c}\text { NICU duration } \\
\text { (days) }\end{array}$} & Mean \pm SD & $2.6 \pm 1.5$ & $4.2 \pm 1.8$ & \multirow{2}{*}{$\wedge 0.069$} & \multirow{2}{*}{--} \\
\hline & Range & $1.0-5.0$ & $2.0-7.0$ & & \\
\hline \multicolumn{2}{|c|}{ MV $(n, \%)$} & $1(0.4 \%)$ & $9(3.8 \%)$ & \&0.011* & $0.20(0.03-1.26)$ \\
\hline \multirow{2}{*}{$\begin{array}{l}\text { MV duration } \\
\text { (days) }\end{array}$} & Mean \pm SD & \multirow{2}{*}{0.5} & $1.1 \pm 0.6$ & \multirow{2}{*}{--} & \multirow{2}{*}{--} \\
\hline & Range & & $0.5-2.0$ & & \\
\hline
\end{tabular}

${ }^{\wedge}$ Independent t-test, \#Chi square test*Significant, RR: relative rate, CI: confidence interval 5).

The neonatal mortality was non-significantly less frequent among betamethasone group (Table

Table 6: comparison between the studied groups regarding neonatal mortality

\begin{tabular}{|c|c|c|c|c|}
\hline Condition & Betamethasone (N=239) & Dexamethasone (N=236) & P & $\begin{array}{c}\text { RR } \\
(\mathbf{9 5 \%} \text { CI) }\end{array}$ \\
\hline Died & $1(0.4 \%)$ & $3(1.3 \%)$ & \multirow{2}{*}{$\& 0.370$} & $\begin{array}{c}0.49 \\
(0.09-2.71)\end{array}$ \\
\hline Lived & $238(99.6 \%)$ & $233(98.7 \%)$ & \\
\hline
\end{tabular}

\&Fisher's Exact test

\section{DISCUSSION}


Preterm birth is the most costly complication of pregnancy and the leading cause of neonatal morbidity and mortality. There are multiple strategies to minimize the risk and the impact of prematurity, such as administration of antenatal corticosteroids ${ }^{(8)}$.When infants were delivered near or at term especially by elective caesarean delivery before the onset of spontaneous vaginal delivery, usually they are deprived of necessary hormonal changes resulting in the development respiratory complications especially neonatal respiratory distress syndrome ${ }^{(6)}$. Caesarean section carried out before the onset of labor is considered to increase the risk of RDS the incidence of respiratory distress syndrome was approximately $0.37 \%$ among those neonates delivered by elective caesarean section ${ }^{(5)}$. Antenatal corticosteroid therapy were thought to improve surfactant production and there was also an associated reduction in the risk of neonatal intraventricular hemorrhage, necrotizing enterocolitis, retinopathy of prematurity, neonatal death and infant mortality by $(30 \%)$, neonatal respiratory distress syndrome by $(50 \%)$, and of both intracranial hemorrhage and periventricular leukomalacia by $(70 \%)$ the later 2 conditions are among the best predictors of long term neurodevelopment injury, including cerebral palsy ${ }^{(9)}$. Both betamethasone and dexamethasone were recommended for use before delivery by American national institutes of Health ${ }^{(\mathbf{1 0})}$. Both betamethasone and dexamethasone were cross the placenta and are not inactivated to a major extent by placental enzymes. Both drugs have a fluoride substituted in the steroid, greatly increasing glucocorticoid potency and giving negligible mineralocoticoid effect ${ }^{(11)}$. The question remains why betamethasone and dexamethasone exert different action in the human fetus. Betamethasone and dexamethasone are stero isomers with the only molecular difference being the c-16 methyl group in the alpha or beta position, respectively. They both readily cross the placenta and the fetal plasma levels of betamethasone and dexamethasone are usually $30 \%$ of maternal plasma levels ${ }^{(\mathbf{1 0})}$. The affinity of betamethasone for the glucocorticoid receptor is higher than that of dexamethasone (7.1 and 5.4 times the affinity of cortisol, respectively). Therefore, the plasma half-life of dexamethasone is shorter than that of betamethasone in the adult $(3.0 \mathrm{~h}$ and $5.6 \mathrm{~h}$, respectively) and human fetus, although the biological half-life is glucocorticoid receptor and the longer plasma half-life of betamethasone had more pronounced effects than exposure to dexamethasone ${ }^{(9)}$.This was prospective randomized study that was conducted at El Hussein University Hospitals on 500 pregnant women who were selected for elective caesarean delivery at (37-37+6weeks) to assess the comparison between betamethasone and dexamethasone administration in reducing respiratory morbidity and mortality. The objective of this study was to assess the comparison between betamethasone and dexamethasone as prophylactic corticosteroid administration 48 hours before the elective caesarian as regard reducing neonatal respiratory morbidity and admission to special care units with respiratory complications. All cases performed elective caesarean section at term (between 37wk and 37+6wks), their neonatal outcomes were examined for any respiratory or non-respiratory morbidity and those results were analyzed by SPSS program.

The recruited 500 women were randomized into two groups:

- Group I [betamethasone Group] ( $\mathrm{n}=250)$ : including women who received intramuscular betamethasone $12 \mathrm{mg} \quad(2$ doses, 24 hours apart) 48 hours before performing the CS.

Lost of follow up $(n=11)$ and analyzed $(\mathrm{n}=239)$.

- Group II [dexamethasone Group] $(\mathrm{n}=250)$ : including women who received intramuscular dexamethasone $8 \mathrm{mg}$ (3 doses, 8 hours apart) 48 hours before performing the CS.

Lost of followup ( $n=14)$ and analyzed $(n=236)$.

No significant difference between the studied groups regarding maternal characteristics but regarding the indication for the C.S, there was no significant difference between women of both groups, however it appears from the data that $434(91.3 \%)$ of the caesarean deliveries were due to previous C.S., which gives an idea about the leading cause for increased caesarean delivries.In the current study there was no significant difference between women of both groups regarding neonatal gender. In contrast to Crowther et al. (12) who tried repeated doses of prenatal corticosteroids to reduce the occurrence and severity of neonatal lung disease and the risk of 
serious health problems in the first few weeks of life. These short-term benefits for babies support the use of repeat dose(s) of prenatal corticosteroids for women at risk of preterm birth. However, these benefits are associated with a reduction in some measures of weight, and head circumference at birth, and there is still insufficient evidence on the longer-term benefits and risk. Also, in another study done by Balci et al. ${ }^{(13)}$ who has done a study on 100 pregnant women in their $24^{\text {th }}-34^{\text {th }}$ weeks of pregnancy who were diagnosed as susceptible to have preterm delivery. Fifty patients were received dexamethasone (Group 1). The other 50 patients were administered $12 \mathrm{mg}$ betamethasone in a single dose (group 2). They found that group 2 babies had better Apgar scores when compared to group 1, and the difference was statistically significant and the study included that administration of a single dose of betamethasone to pregnant women in their $24^{\text {th }}-34^{\text {th }}$ weeks of pregnancy who are likely to have preterm delivery reduces RDS development. In the current study, the rate of neonatal respiratory distress syndrome was lower in group I [betamethasone group] when compared to group II [dexamethasone group] $[1 / 239(0.4 \%)$ vs. $7 / 236(3.0 \%)$, respectively] as evidenced by lower Down score which was statistically significant with $\mathrm{p}$ value $=0.031$. Feldman et al. ${ }^{(14)}$ this is in agreement with the previous results in which the most pronounced finding in their study was the lower rate of RDS among neonates who were exposed to betamethasone than dexamethason.(RDS (\%) $62.9 \%$ vs. $81.8 \%$ ) respectively which statistically highly significant with $\mathrm{p}$ value $=$ 001. Elimian et al. (4) disagreement with the previous results claiming that for respiratory distress no difference was seen between those infants exposed to betamethasone compared with exposure to dexamethasone. Also Brownfoot et al. ${ }^{(15)}$ systematic review in five trials that made a direct comparison in the (RR $1.06,95 \%$ CI 0.88 to $1.27,5$ trials, $\mathrm{n}=753$ ) which reveal that there is no significant difference regarding the incidence of RDS in both betamethasone and dexamethasone. Regarding TTN our study showed that the incidence of TTN in group I [betamethasone Group] was statistically significant when compared to group II [dexamethasone Group] [4/239 (1.7\%) vs. 16/236 (6.8\%), respectively] which was statistically significant with $\mathrm{p}$ value $=$ 0.006 . This agree with Stutchfield $\boldsymbol{e t} \boldsymbol{a l} .{ }^{(16)}$ who published a provocative article showing a reduction in the incidence of transient tachypnoea (TTN) in infants of mothers submitted to elective caesarean section receiving antenatal betamethasone.In another study carried out by James et al. ${ }^{(17)}$ they believed that surfactant deficiency and/or dysfunction is a major determinant of respiratory distress in term infants diagnosed as having TTN and that enhanced surfactant production by betamethasone may explain its reduced incidence of TTN. Low levels of phosphatidylglycerol in the lung fluid and in amniotic fluid were reported in patients with TTN. Also our study revealed that the need for neonatal resuscitation was significantly lower in group I [betamethasone Group] when compared to group II [dexamethasone Group] [6/239 (2.5\%) vs. $28 / 236(11.9 \%)$, respectively] which was statistically significant with $\mathrm{p}$ value $=0.001$. In the current study, the rate of admission to neonatal intensive care unit (NICU) for respiratory morbidity was significantly lower in group I [betamethasone Group] when compared to group II [Dexamethasone Group] [5/239(2.1\%) vs. $25 / 236(10.6 \%)$ ] respectively which was statistically significant with $\mathrm{p}$ value $=0.001 . \mathrm{n}$ one trial of 105 infants, significantly more infants in the dexamethasone group were admitted to NICU compared with the betamethasone group (RR 3.83, 95\% CI 1.24 to 11.87) which consistent with our study ${ }^{(15)}$.While, Danesh et al. ${ }^{(18)}$ reported that the number of infants admitted to the neonatal intensive care unit (NICU) for women with intactmembranes; 9/60 in the dexamethasone group was admitted versus $12 / 60$ in the betamethasone group.In the present study, regarding the rate of the need for mechanical ventilation only one $[1 / 239(0.4 \%)$ patient in betamethasone group needed MV when compared to $9[9 / 239(3.8 \%)]$ patients in dexamethasone group which was statistically significant with $\mathrm{p}$ value $=0.011$. Roberts and Dalziel ${ }^{(\mathbf{1})}$ showed that treatment with antenatal corticosteroids at term was associated with less need to neonatal respiratory support; with a reduction in the need for mechanical ventilation, continuous positive airway pressure (CPAP). Less time requiring oxygen supplementation but with no statistically significant difference between the intervention and control groups. The neonatal mortality was non-significantly less frequent among group I 
[betamethasone Group] when compared to group II [Dexamethasone Group] [1/239(0.4\%) vs. 3/236(1.3\%)] respectively which was statistically non-significant with $\mathrm{p}$ value $=0.370$, but a National Institute of Child Health and Human Development Neonatal Research Network (NICHD NRN) cohort study of over 300 infants reported a link between betamethasone and reduced risk of neonatal death whereas dexamethasone was associated with an increased risk of neonatal death ${ }^{(\mathbf{1 9})}$. In contrast, the Roberts et al. ${ }^{(1)}$ Cochrane review showed a reduced risk for fetal and neonatal death for both the betamethasone and dexamethasone groups compared with placebo/no treatment. In a later NICHD NRN report of part of this cohort, Lee et al. (21) reported reduced adverse childhood neurological outcomes at 18 to 22 months for dexamethasone, but not for betamethasone. Also, Feldman et al. ${ }^{(14)}$ found that the rate of neonatal death was similar in each group with $\mathrm{p}$ value $=0.79$.

\section{CONCLUSION}

Antenatal Corticosteroids (ecpecially betamethasone) are effective in prevention of respiratory complications after elective caesarean section at term including:

1. The incidence of admission with respiratory distress to neonatal ICU.

2. The incidence of admission to primary care baby unit (PCBU).

3. The incidence of transient tachyponea of the newborn.

\section{REFERENCES}

1. Roberts D and Dalziel S (2017): Antenatal corticosteroids for accelerating fetal maturation for women at risk of preterm birth. Syst. Rev. Cochrane Database, 3: 4454.

2. Mwansa-Kambafwile $J$, Cousens $\mathrm{S}$, Hansen T et al. (2010): Antenatal steroids in preterm labour for the prevention of neonatal deaths due to complications of preterm birth. Int. J. Epidemiol., 39(I): 122-133.

3. American College of Obstetrician and Gynaecologist (ACOG) (2011): Antenatal corticosteroid therapy for fetal maturation. Obstet. Gynecol., 99: 871873.

4. Elimian A, Garry D, Figueroa $\mathrm{R}$ et al. (2007): Antenatal betamethasone compared with dexamethasone (Betacode Trial): a randomized controlled trial. Obstetrics and Gynecology, 110(1): 2630.

5. Royal College of Obstetricians and Gynaecologists (RCOG) (2010): Antenatal corticosteroids to reduce neonatal morbidity and mortality. Greentop Guideline, https://www.glowm.com/.../Antenatal\%2 0Corticosteroids\%20to\%20Reduce $\% 20 \mathrm{~N}$ eona

6. Morrison JJ, Rennie JM and Milton PJ (1995): Neonatal respiratory morbidity and mode of delivery at term: influence of timing of elective caesarean section. Obstet. Gynaecol., 102: 101-106.

7. Downes JJ, Vidyasagar D, Morrow GM et al. (1970): Respiratory distress syndrome of newborn infant. Clin. Pediatr., 9(6): 325-331.

8. Kuk JY, An JJ, Cha HH et al. (2013): Optimal time interval between a single course of antenatal corticosteroids and delivery for reduction of respiratory distress syndrome in preterm twins. Am. J. Obstet. Gynecol., 209(3): 256-266.

9. Shane E, Burr D and Abrahamsen B (2014): Atypical subtrochanteric and diaphyseal femoral fractures. J. Bone Miner. Res., 29: 1-23.

10. National Institutes of Health (NIH) (1995): Consensus development panel on the effect of corticosteroids for fetal maturation on perinatal outcomes. National Institute of Health, Bethesda, Maryland. JAMA., 273: 413-418.

11. Jobe AH and Soll RF (2014): Choice and dose of corticosteroid for antenatal treatments. Am. J. Obstet. Gynecol., 190(4): 878-881.

12. Crowther CA and Harding JE (2008): Repeat doses of prenatal corticosteroids for women at risk of preterm birth for preventing neonatal respiratory disease. Cochrane Database of Systematic Reviews, 3: 3935-3942.

13. Balci O, Ozdemir S, Mahmoud AS et al. (2010): The effect of antenatal steroids on fetal lung maturation between the 24th and 34th week of pregnancy. Gynecol. Obstet. Investigations, 70(2): 95-99.

14. Feldman DM, Carbone J, Belden L et al. (2007): Betamethasone vs dexamethasone for the prevention of morbidity in very- 
lowbirthweight neonates. Am. J. Obstet .Gynecol., 197: 284-293.

15. Brownfoot FC, Crowther $\mathrm{CA}$ and Middleton P (2013): Different corticosteroids and regimens for accelerating fetal lung maturation for women at risk of preterm birth. Cochrane Database of Systematic Reviews, doi: 10.1002/14651858

16. Stutchfield PR, Whittaker $R$ and Russell (2012): Antenatal betamethasone and incidence of neonatal respiratory distress after elective caesarean section; pragmatic randomised trial. BMJ., 331: 662-664.

17. James DK, Chiswick ML and Harces A (2010): Non- specificity of surfactant deficiency in neonatal respiratory disorders. Br Med J., 288: 1635-1638.

18. Danesh A, Janghorbani $M$ and Khalatbari S (2012): Effects of antenatal corticosteroids on maternal serum indicators of infection in women at risk for preterm delivery: A randomized trial comparing betamethasone and dexamethasone. Journal of Research in Medical Sciences, 17(10): 911-917.

19. Lee BH, Stoll BJ, McDonald SA et al. (2006): For the National Institute of Child Health and Human Development Neonatal Research Network Adverse neonatal outcomes associated with antenatal dexamethasone versus antenatal betamethasone. Pediatrics, 117(5): 15031510.

20. Roberts D and Dalziel S (2006): Antenatal corticosteroids for accelerating fetal lung maturation for women at risk of preterm birth. Cochrane Database of Systematic Reviews, 3(19): 44544462-.

21. Lee BH, Stoll BJ, McDonald SA et al. (2008): For the National Institute of Child Health and Human Development Neonatal Research Network. Neurodevelopmental outcomes of extremely low birth weight infants exposed prenatally to dexamethasone versus betamethasone. Pediatrics, 121(2): 289-296. 